\title{
EFFECTIVE INSEPARABILITY FOR SEQUENCES OF SETS
}

\author{
A. H. LACHLAN
}

The notions of effective inseparability (for pairs of sets) and of double productivity are defined in [5]. In this paper are considered some extensions of these notions for sequences of sets, and also certain alternative formulations of them in the spirit of Mučnik [3].

Let $M$ be a nonempty recursively enumerable (r.e.) set which will be fixed in each context. By a sequence of sets we mean a sequence of sets of natural numbers indexed by $M$; by a disjoint sequence we mean one whose members are pairwise disjoint. Our suffixes will range over $M$, thus for a sequence of sets we shall use a notation such as $\left\{A_{x}\right\} ; A_{x}^{*}$ will be used to denote the set of numbers which belong to $A_{x}$ but not to any other member of the sequence $\left\{A_{x}\right\}$. The complement of a set $A$ will be denoted by $A^{\prime}$. We use $\bigvee_{x}$ and $\Lambda x$ as universal and existential quantifiers over $M$. Superscripts will range over the set $N$ of all natural numbers; variables left free should be interpreted as universally quantified over $N$, or over $M$ for suffixes.

A sequence of sets $\left\{A_{x}\right\}$ is called r.e. if there is a binary r.e. relation $A(x, y)$ such that

$$
x \in M \rightarrow\left(A(x, y) \leftrightarrow y \in A_{x}\right) .
$$

We may suppose that $A(x, y)$ fails for $x \notin M$, because $M$ is r.e. The idea of a standard enumeration is developed in [2]; let $\left\{W^{z}\right\}, z=0,1$, $2, \cdots$ be a standard enumeration of the r.e. sets, and let $\left\{W_{x}^{z}\right\}$, $z=0,1,2, \cdots$ be a standard enumeration of the r.e. sequences of sets. We choose a particular effective way of simultaneously enumerating all the sets $W_{x}^{z}$ and define

$$
\begin{aligned}
D_{x}^{z}= & \left\{y \mid y \text { turns up in } W_{x}^{z}\right. \text { before it turns up in any other member } \\
& \text { of } \left.\left\{W_{x}^{z}\right\}\right\},
\end{aligned}
$$

then we have

$$
D_{x}^{2} \subseteq W_{x}^{z} \text { and } \bigcup_{x} D_{x}^{z}=\bigcup_{x} W_{x}^{2}
$$

and $\left\{D_{x}^{2}\right\}, z=0,1,2, \cdots$ is a standard enumeration of the standard class consisting of the disjoint r.e. sequences.

A sequence of sets $\left\{A_{x}\right\}$ is said to be $m$-reducible to a sequence $\left\{B_{x}\right\}$

Received by the editors April 1, 1964. 
if there is a recursive function $\phi$ such that

$$
\bigwedge x\left(y \in A_{x} \leftrightarrow \phi(y) \in B_{x}\right) .
$$

We call a sequence $\left\{A_{x}\right\}$ universal (for disjoint sequences) if every disjoint r.e. sequence is $m$-reducible to $A_{x}$.

Smullyan's results pertain to the case $M=\{0,1\}$, in which case the sequences of sets are called pairs of sets.

A sequence $\left\{A_{x}\right\}$ is called coproductive if there exists a recursive function $\alpha$ such that

$$
\bigwedge x\left(W_{x}^{z} \cap A_{x}^{*}=\varnothing\right) \rightarrow{ }_{x}\left(\alpha(z) \notin W_{x}^{z} \cup A_{x}\right) .
$$

Similar definitions have been given by Cleave (definition of creative sequence, $[1$, p. 206]) and by Smullyan (definition of doubly productive, [5, p. 107]). Each of those authors proved the version of the following theorem corresponding to his definition:

TheOREM 1. The sequence $\left\{A_{x}\right\}$ is coproductive iff it is universal.

Proor. Let $\alpha$ be a recursive function satisfying (1), and let $\left\{S_{x}\right\}$ be any disjoint r.e. sequence. We define

$$
T_{x}^{m, n}= \begin{cases}\alpha(n) & \text { if } m \in S_{x} \\ \varnothing & \text { otherwise }\end{cases}
$$

by the recursion theorem, in the shape of Corollary 2.2 of [2], there is a recursive function $\beta$ such that

$$
T_{x}^{m, \beta(m)}=W_{x}^{\beta(m)} .
$$

It now follows from (1) that

$$
y \in S_{x} \leftrightarrow \alpha \beta(y) \in A_{x},
$$

and so $\left\{A_{x}\right\}$ is universal.

Conversely, suppose that $\left\{A_{x}\right\}$ is universal, then it follows by the recursion theorem that there is a recursive function $\alpha$ such that

$$
\alpha(z) \in D_{x}^{z} \leftrightarrow \alpha(z) \in A_{x} .
$$

For this application we transpose our sequences of sets, which by convention are here indexed by $M$, into sequences of subsets of $M$ indexed by $N$; we then apply Corollary 2.2 of [2] again with $\subseteq=\varsigma_{M}$. From (2) and the definition of $D_{x}^{z}$ the truth of (1) follows easily. This completes the proof.

If $M$ has cardinality $>1$ and if the sequence $\left\{A_{x}\right\}$ is r.e., then the 
condition (1) can be varied in several ways so that it remains equivalent to universality. For instance, an r.e. sequence $\left\{A_{x}\right\}$ is called effectively inseparable if there is a recursive function $\alpha$ such that

$$
\begin{aligned}
& \wedge x \wedge y\left(W_{x}^{2} \cap A_{x}^{*}=\varnothing \wedge\left(x \neq y \rightarrow: W_{x}^{z} \cap W_{y}^{2} \subseteq \bigcup_{t} A_{t} \wedge W_{x}^{2} \supseteq A_{y}\right)\right) \\
& \rightarrow \wedge x\left(\alpha(z) \notin W_{x}^{z}\right) .
\end{aligned}
$$

This generalizes Smullyan's definition, [5, p. 120]. Following Smullyan we show that effective inseparability implies coproductiveness, the converse being immediate. Assume therefore that $\alpha$ is a recursive function satisfying (3) and define

$$
T_{x}^{z}=\left\{y \mid y \in D_{x}^{z} \vee \bigvee w\left(w \neq x \wedge y \in A_{w}\right)\right\}
$$

Since $\left\{A_{x}\right\}$ is assumed r.e., the relation $y \in T_{x}^{z}$ between $x, y, z$ is r.e., and so there exists a recursive function $\beta$ such that $T_{x}^{z}=W_{x}^{\beta(z)}$. Further, if the 1.h.s. of (1) holds, then the 1.h.s. of (3) holds with $z$ replaced by $\beta(z)$. Hence we have

$$
\bigwedge x\left(\alpha \beta(z) \notin W_{x}^{\beta(z)}\right)
$$

However, assuming that the cardinality of $M$ is $>1$, we have

$$
\bigcup_{x} W_{x}^{\beta(z)}=\bigcup_{x}\left(W_{x}^{z} \cup A_{x}\right) \text {. }
$$

Thus, if the 1.h.s. of (1) holds, the r.h.s. of (1) holds with $\alpha$ replaced by $\alpha \beta$. We have proved

LeMma 1. An r.e. sequence is coproductive iff it is effectively inseparable, provided that $M$ has cardinality $>1$.

There are other ways in which we can frame a condition equivalent to universality. We can write ' $D$ ' for ' $W$ ' throughout (1). If $\left\{A_{x}\right\}$ is r.e. and the cardinality of $M$ is $>1$, we can clearly omit the final $A_{x}$ from (1). Further, in each variation we only require $\alpha$ partial recursive (p.r.) and defined at least when the 1.h.s. of the relevant implication holds.

In [3] Mučnik proved that an r.e. set $C$ is complete if there is a recursive function $\alpha$ such that

$$
W^{z} \cup C=N \rightarrow \alpha(z) \in W^{z} \cap C .
$$

The author has noticed that this result and its proof can be obtained by taking complements in the earlier result of Myhill that a creative set with recursive productive function is complete. In fact, the dual- 
ity can be carried a little further: call a singulary function $\phi d u a l$ p.r. if the relation $\phi(x) \neq y$ is r.e., then we have

Lemma 2. The set $A$ is universal iff there is a dual p.r. function $\alpha$ such that

$$
W^{*} \cup C=N \rightarrow\left(\alpha(z) \text { defined } \wedge \alpha(z) \in W^{z} \cap A\right) .
$$

Here 'universal' means universal as a sequence of length one, and the proof of the lemma can be got from that of our next theorem.

The problem naturally arises of finding a dual formulation of Theorem 1; this is not trivial because in Theorem 1 we are no longer dealing with sets but with sequences. We propose as dual to (1) the implication

$$
\bigvee x\left(W_{x}^{z} \cup A_{x}=N\right) \rightarrow \bigvee x\left(\alpha(z) \in W_{x}^{z} \cap A_{x}^{*}\right) .
$$

We now have as the dual of Theorem 1 :

THEOREM $1^{\prime}$. The sequence $\left\{A_{x}\right\}$ is universal iff there is a recursive function $\alpha$ satisfying $\left(1^{\prime}\right)$.

Carrying the proof of the 'if' part over from Theorem 1, the key step is the definition:

$$
T_{x}^{m, n}=\left\{\begin{array}{l}
N-\{\alpha(n)\} \quad \text { if } m \notin S_{x}, \\
N \text { otherwise. }
\end{array}\right.
$$

The 'only if' part follows straight from (2) as before.

A sequence $\left\{E_{x}\right\}$ is called dual disjoint if the sequence $\left\{E_{x}^{\prime}\right\}$ is disjoint. Notice that $\left(1^{\prime}\right)$ is only required for $z$ such that $\left\{W_{x}^{2}\right\}$ is dual disjoint. There seems to be no perfect analogy to (3), the nearest we can get is

$$
\begin{aligned}
\bigvee x\left(W_{x}^{2} \cup A_{x}=N\right) \wedge & \wedge x \wedge y\left(x \neq y \rightarrow W_{x}^{2} \supseteq A_{y}\right) \\
& : \rightarrow \bigvee x\left(\alpha(z) \in W_{x}^{2} \cap A_{x}^{*}\right) .
\end{aligned}
$$

This is shown equivalent to $\left(1^{\prime}\right)$ for an r.e. sequence $\left\{A_{x}\right\}$ by an argument similar to that used for Lemma 1 . Also, we can substitute 'dual p.r. function' for 'recursive function' in Theorem 1' (or its variant) provided that we add the conjunctand ' $\alpha(z)$ defined' on the r.h.s. of the corresponding implication.

It is interesting to note that, when $\left\{A_{x}\right\}$ is r.e. and the cardinality of $M$ is $>1$, then the initial $A_{x}$ can be omitted from ( $\left(1^{\prime}\right)$ just as the final $A_{x}$ can be omitted from (1). We alter the definition of $T_{x}^{m, n}$ to 


$$
T_{x}^{m, n}=\left\{\begin{array}{l}
N \quad \text { if } m \in S_{x} \vee \bigvee y\left(y \neq x \wedge \alpha(n) \in A_{y}\right), \\
N-\{\alpha(n)\} \quad \text { otherwise. }
\end{array}\right.
$$

This modification is required so that we can prove the implication $\Lambda_{x}\left(m \notin S_{x}\right) \rightarrow \Lambda_{x}\left(\alpha \beta(m) \notin A_{x}\right)$, where $\beta$ is defined as in Theorem 1 . This alteration enables us to prove the 'if' part of Theorem $1^{\prime}$ ' when $A_{x}$ is omitted from $\left(1^{\prime}\right)$. Since the omission weakens $\left(1^{\prime}\right)$, the 'only if' part is immediate.

There is one result of Mučnik (Theorem 5 of [3]) which is not included in any of the results given above. We now prove two theorems from both of which Mučnik's theorem can be obtained by taking $M=\{0,1\}$ and $\left\{A_{x}\right\}$ to be a disjoint r.e. pair.

THEOREM 2. Let $M$ have cardinality $>1$. An r.e. disjoint sequence $\left\{A_{x}\right\}$ is universal iff there is a recursive function $\alpha$ such that

$$
\bigcup_{x} W_{x}^{2}=N \wedge \wedge x \Lambda_{y}\left(x \neq y \rightarrow W_{x}^{2} \supseteq A_{y}\right): \rightarrow \Lambda_{x}\left(\alpha(z) \in W_{x}^{2}\right) .
$$

Proof. For the 'if' part suppose that $\alpha$ is a recursive function satisfying (4) and that $\left\{S_{x}\right\}$ is any disjoint r.e. sequence. We now use precisely the same construction for $T_{x}^{m, n}$ which we used to justify the omission of $A_{x}$ from ( $\left(1^{\prime}\right)$. We then apply the recursion theorem as in Theorem 1 to obtain a recursive function $\beta$ such that

$$
T_{x}^{m, \beta(m)}=W_{x}^{\beta(m)} .
$$

If $\alpha \beta(m) \in A_{x}$, then the 1.h.s. of (4) is satisfied for $z=\beta(m)$, whence $\alpha \beta(m)$ is in every member of $\left\{W_{t}^{\beta(m)}\right\}$, whence $m \in S_{x}$ from the definition of $T_{x}^{m, \beta(m)}$. Conversely, if $m \in S_{x}$, then the 1.h.s. of (4) again holds for $z=\beta(m)$, whence $\alpha \beta(m)$ is in every member of $\left\{W_{t}^{\beta(m)}\right\}$, whence $\alpha \beta(m)$ is in $A_{x}$ from the definition of $T_{x}^{m, \beta(m)}$.

For the 'only if' part we use (2) once again. If $\left\{W_{x}^{2}\right\}$ covers $N$, so does $\left\{D_{x}^{z}\right\}$. Hence $\alpha(z)$ is in $D_{i}^{z}$ for some $i$, and from (2) we have $\alpha(z) \in A_{i}$. If the second conjunctand on the 1.h.s. of (4) holds (as well as the first), then $\alpha(z) \in W_{y}^{2}$ for all $y, y \neq i$. Thus (4) holds for the function $\alpha$ which satisfies (2).

Theorem 3. Let $M$ have finite cardinality $>1$. An r.e. disjoint sequence $\left\{A_{x}\right\}$ is universal iff there is a recursive function $\alpha$ such that

$$
\left\{W_{x}^{z}\right\} \text { is dual disjoint } \wedge \wedge_{x}\left(W_{x}^{2} \supseteq A_{x}\right): \rightarrow \Lambda x\left(\alpha(z) \in W_{x}^{z}\right) .
$$

Proof. Let $\alpha$ be a recursive function satisfying (5) and let $\left\{S_{x}\right\}$ be a disjoint r.e. sequence. Let $\rho$ be a function which permutes the members of $M$ cyclically. Given $m, n$, we carry out simultaneously two 
searches: in search 1 we seek a number $x$ such that $m \in S_{x}$, in search 2 we seek a number $x$ such that $\alpha(n) \in A_{x}$. The first number found in search 1 will be denoted by $\kappa(m)$, the first number found in search 2 will be denoted by $\lambda(n)$. We define $T_{x}^{m, n}$ by

$T_{x}^{m, n}=\left\{\begin{array}{l}N \text { if search } 1 \text { finishes first and } x \neq \kappa(m), \text { or if search } 2 \text { finishes } \\ \quad \text { first and } x \neq \rho \lambda(n), \\ (N-\{\alpha(n)\}) \cup A_{x} \text { otherwise. }\end{array}\right.$

We now define $\beta$ as before and as before we can show that $\alpha \beta$ reduces $\left\{S_{x}\right\}$ to $\left\{A_{x}\right\}$.

For the 'only if' part, define

$$
E_{x}^{2}=\left\{y \mid y \text { turns up in each } W_{t}^{2} \text { with } t \neq x \text { before it turns up in } W_{x}^{2}\right\},
$$

where it is not required that $y$ should turn up in $W_{x}^{2}$ for $y$ to belong to $E_{x}^{z}$. Then, if $\left\{W_{x}^{2}\right\}$ is a dual disjoint sequence, $\left\{E_{x}^{z}\right\}$ is a disjoint sequence covering $N$. By an application of the recursion theorem similar to that which yields (2), we have, assuming that $\left\{A_{x}\right\}$ is universal, a recursive function $\alpha$ such that

$$
\alpha(z) \in E_{x}^{z} \leftrightarrow \alpha(z) \in A_{x} .
$$

From this the implication (5) follows easily. This completes the proof of the theorem.

We can counterexample this theorem for $M$ infinite by supposing that $\alpha$ is a recursive function satisfying (5) for a disjoint r.e. sequence $\left\{A_{x}\right\}$. We construct a sequence $\left\{T_{x}^{n}\right\}$ as follows, supposing without loss of generality that $M=N$. We begin enumerating $N-\{\alpha(n)\}$ in $T_{0}^{n}$ and $N$ in each of the remaining sets of $\left\{T_{x}^{n}\right\}$. However, if $\alpha(n)$ turns up in $A_{0}$, we let $m$ be the least number such that $\alpha(n)$ has not yet been enumerated in $T_{m}^{n}$. We now complete the enumeration so that $T_{m}^{n}=N-\{\alpha(n)\}$ and so that $T_{x}^{n}=N$ for $x \neq m$. We can arrange the details so that $x \in T_{z}^{y}$ is an r.e. relation between $x, y, z$. This entitles us to use the recursion theorem to obtain a number $e$ such that $T_{x}^{e}=W_{x}^{e}$. Considering (5) for $z=e$ we obtain a contradiction, because the 1.h.s. of the implication is satisfied but not the r.h.s.

We can also show that there can be no dual p.r. function $\alpha$, defined at least when the 1.h.s. of (5) holds, which satisfies (5). We use the same technique coupled with the priority ordering method ascribed to Friedberg and Mučnik. For, if $\alpha$ is dual p.r., we can effectively enumerate all numbers $\neq \alpha(n)$. Thus in the construction of $\left\{T_{x}^{n}\right\}$ we first adopt 0 as a provisional $\alpha(n)$; if this becomes untenable, i.e., if 
we enumerate 0 as $\neq \alpha(n)$, then we adopt the least possible number as provisional $\alpha(n)$, and so on. For each provisional $\alpha(n)$ we perform a construction similar to the above, abandoning it for a new construction if a new $\alpha(n)$ has to be adopted. In fixing the details we have only to ensure that, if $\alpha(n)$ is undefined, then $T_{x}^{n}=N$ for all $x$. It is vital to both constructions that after any particular step only a finite number of numbers will have been enumerated in $U_{x} T_{x}^{n}$.

Theorems 2 and 3 hold for nondisjoint r.e. sequences $\left\{A_{x}\right\}$ provided that for the right hand sides of (4) and (5) we substitute

$$
\Lambda_{x} \Lambda_{y}\left(\alpha(z) \in W_{x}^{z} \wedge\left(x \neq y \rightarrow \alpha(z) \notin A_{x} \cap A_{y}\right)\right) .
$$

Further, in each of Theorems 2 and 3 we can substitute 'dual p.r. function' for 'recursive function' if we add ' $\alpha(z)$ defined' on the r.h.s. of (4) and (5).

In conclusion, we remark that the duality notion noted above may well prove interesting in other contexts. For instance, we can consider sets retraced by dual p.r. functions, and dual recursive equivalence types.

\section{REFERENCES}

1. J. P. Cleave, Creative functions, Z. Math. Logik Grundlagen Math. 7 (1961), 205-212.

2. A. H. Lachlan, Standard classes of recursively enumerable sets, Z. Math. Logik Grundlagen Math. 10 (1964), 23-42.

3. A. A. Mučnik, Isomorphism of systems of recursively enumerable sets, Amer. Math. Soc. Transl. (2) 23 (1963), 7-13.

4. J. Myhill, Creative sets, Z. Math. Logik Grundlagen Math. 1 (1955), 97-108.

5. R. M. Smullyan, Theory of formal systems, Annals of Mathematics Studies, No. 47, Princeton Univ. Press, Princeton, N. J., 1961 (rev. ed.).

University of NewCASTLE Upon TyNe, England 